\title{
TURBULENT FIELDS AND THEIR RECURRENCES
}

\author{
PREDRAG CVITANOVIĆ AND YUEHENG LAN \\ Center for Nonlinear Science, School of Physics, \\ Georgia Institute of Technology, Atlanta 30332-0430, U.S.A. \\ E-mail: predrag.cvitanovic@physics.gatech.edu
}

\begin{abstract}
We introduce a new variational method for finding periodic orbits of flows and spatio-temporally periodic solutions of classical field theories, a generalization of the Newton method to a flow in the space of loops. The feasibility of the method is demonstrated by its application to several dynamical systems, including the Kuramoto-Sivashinsky system.
\end{abstract}

\section{Introduction}

Chaos is the norm for generic Hamiltonian flows, and for path integrals that implies that instead of a few, or countably many extremal configurations, classical solutions populate fractal sets of saddles. For the path-integral formulation of quantum mechanics such solutions were discovered by Gutzwiller 1 who derived a trace formula that relates a semi-classical approximation of the energy eigenspectrum to the classical periodic solutions. While the theory has worked very well in quantum mechanical applications, these ideas remain largely unexplored in quantum field theory.

The classical solutions for most strongly nonlinear field theories are nothing like the harmonic oscillator degrees of freedom, the electrons and photons of QED; they are unstable and highly nontrivial, accessible only by numerical techniques. The new aspect, prerequisite to a semi-classical quantization of strongly nonlinear field theories, 2 is the need to determine a large number of spatio-temporally periodic solutions for a given classical field theory. Why periodic?

The dynamics of strongly nonlinear classical fields is turbulent, not "laminar", and how are we to think about turbulent dynamics? Hopf 3 and Spiegel 4156 have proposed that the turbulence in spatially extended systems be described in terms of recurrent spatiotemporal patterns. Pictorially, dynamics drives a given spatially extended system through a repertoire of unstable patterns; as we watch a turbulent system evolve, every so often we catch a glimpse of a familiar pattern. For any finite spatial resolution, for a finite time the system follows approximately a pattern belonging to a finite alphabet of admissible patterns, and the long term dynamics can be thought of as a walk through the space of such patterns, just as chaotic dynamics with a low dimensional attractor can be thought of as a succession of nearly periodic (but unstable) motions. So periodic solutions are needed both to quantify "turbulence" in classical field theory, and as a starting point for the semi-classical quantization of a quantum field theory.

There is a great deal of literature on numerical periodic orbit searches. Here we take as the starting point Cvitanović et al. webbook, 18 and in Sec. 2 briefly review the Newton-Raphson method for low-dimensional flows described by ordinary differential equations (ODEs), in order to motivate the Newton descent approach that

crete02: submitted to World Scientific on March 10, 2003 
we shall use here, and show that it is equivalent to a cost function minimization method.

The problem one faces with high-dimensional flows is that their topology is hard to visualize, and that even with a decent starting guess for a point on a periodic orbit, methods like the Newton-Raphson method are likely to fail. In Sec. 3 we describe a new method for finding spatio-temporally periodic solutions of extended, infinite dimensional systems described by partial differential equations (PDEs), and in Sec. 4 we discuss a simplification of the method specific to Hamiltonian flows.

The idea is to make an informed rough guess of what the desired periodic orbit looks like globally, and then use variational methods to drive the initial guess toward the exact solution. Sacrificing computer memory for robustness of the method, we replace a guess that a point is on the periodic orbit by a guess of the entire orbit. And, sacrificing speed for safety, we replace the Newton-Raphson iteration by the Newton descent, a differential flow that minimizes a cost function computed as deviation of the approximate flow from the true flow along a smooth loop approximation to a periodic orbit.

In Sec. [5 the method is tested on several systems, both infinite-dimensional and Hamiltonian, and its virtues, shortcomings and future prospects are discussed in Sec. [6]

\section{Periodic orbit searches}

A periodic orbit is a solution $(x, T), x \in \mathbb{R}^{d}, T \in \mathbb{R}$ of the periodic orbit condition

$$
f^{T}(x)=x, \quad T>0
$$

for a given flow or mapping $x \rightarrow f^{t}(x)$. Our goal here is to determine periodic orbits of flows defined by first order ODEs

$$
\frac{d x}{d t}=v(x), \quad x \in \mathcal{M} \subset \mathbb{R}^{d}, \quad(x, v) \in \mathbf{T} \mathcal{M}
$$

in many (even infinitely many) dimensions $d$. Here $\mathcal{M}$ is the phase space (or state space) in which evolution takes place, $\mathbf{T} \mathcal{M}$ is the tangent bundle, 9 and the vector field $v(x)$ is assumed smooth (sufficiently differentiable).

A prime cycle $p$ of period $T_{p}$ is a single traversal of the orbit. A cycle point of a flow which crosses a Poincaré section $n_{p}$ times is a fixed point of the $f^{n_{p}}$ iterate of the Poincaré section return map $f$, hence one often refers to a cycle as a "fixed point". By cyclic invariance, stability eigenvalues and the period of the cycle are independent of the choice of an initial point, so it suffices to solve Eq. (1) at a single cycle point. Our task is thus to find a cycle point $x \in p$ and the shortest time $T_{p}$ for which Eq. (10) has a solution.

If the cycle is an attracting limit cycle with a sizable basin of attraction, it can be found by integrating the flow for sufficiently long time. If the cycle is unstable, simple integration forward in time will not reveal it, and methods to be described here need to be deployed. In essence, any method for solving numerically the periodic orbit condition $F(x)=x-f^{T}(x)=0$ is based on devising a new dynamical system which possesses the same cycle, but for which this cycle is attractive. Beyond

crete02: submitted to World Scientific on March 10, 2003 


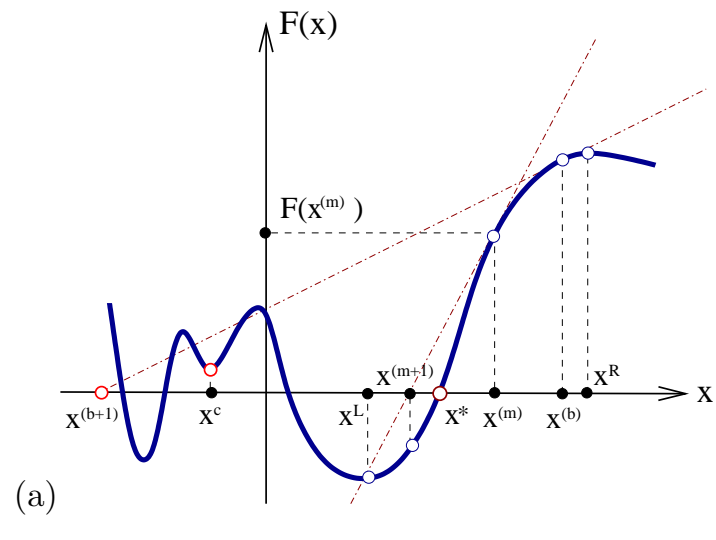

(b)

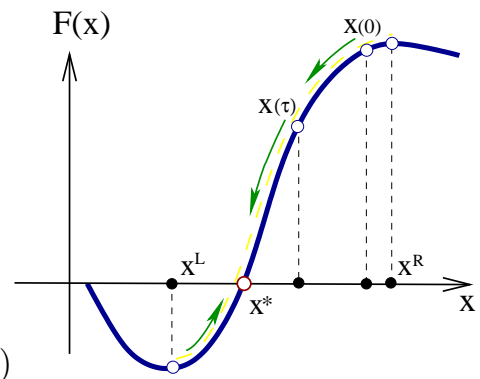

Figure 1. (a) Newton method: bad initial guess $x^{(b)}$ leads to the Newton estimate $x^{(b+1)}$ far away from the desired zero of $F(x)$. Sequence $\cdots, x^{(m)}, x^{(m+1)}, \cdots$, starting with a good guess converges super-exponentially to $x^{*}$. (b) Newton descent: any initial guess $x(0)$ in the monotone interval $\left[x^{L}, x^{R}\right]$ of $F(x)$ flows to $x^{*}$ exponentially fast. Both methods diverge if they fall into the basin of attraction of a local minimum $x^{c}$.

that, there is a great freedom in constructing such systems, and many different methods are used in practice.

\subsection{Newton Descent in 1 Dimension}

Newton's method for determining a zero $x^{*}$ of a function $F(x)$ of one variable is based on a linearization around a starting guess $x^{(0)}$ :

$$
F(x) \approx F\left(x^{(0)}\right)+F^{\prime}\left(x^{(0)}\right)\left(x-x^{(0)}\right) .
$$

An improved approximate solution $x^{(1)}$ of $F(x)=0$ is then $x^{(1)}=x^{(0)}-$ $F\left(x^{(0)}\right) / F^{\prime}\left(x^{(0)}\right)$. Provided that the $m$ th guess is sufficiently close to $x^{*}$, the Newton iteration

$$
x^{(m+1)}=x^{(m)}-F\left(x^{(m)}\right) / F^{\prime}\left(x^{(m)}\right)
$$

converges to $x^{*}$ super-exponentially fast (see Fig. 囵(a)). In order to avoid jumping too far from the desired $x^{*}$, one often initiates the search by the damped Newton method,

$$
\Delta x^{(m)}=x^{(m+1)}-x^{(m)}=-\frac{F\left(x^{(m)}\right)}{F^{\prime}\left(x^{(m)}\right)} \Delta \tau, \quad 0<\Delta \tau \leq 1,
$$

takes small $\Delta \tau$ steps at the beginning, reinstating to the full $\Delta \tau=1$ jumps only when sufficiently close to the desired $x^{*}$.

Let us now take the extremely cautious approach of keeping all steps infinitesimally small, and replacing the discrete sequence $x^{(m)}, x^{(m+1)}, \ldots$ by the fictitious time $\tau$ flow $x=x(\tau)$ :

$$
d x=-\frac{F(x)}{F^{\prime}(x)} d \tau, \quad \tau \in[0, \infty]
$$


If a simple zero, $F^{\prime}\left(x^{*}\right) \neq 0$, exists in any given monotone lap of $F(x)$, it is the attractive fixed point of the flow Eq. (5) (see Fig. 1 (b)).

While reminiscent of "gradient descent" methods, 710 this is a flow, rather than an iteration. For lack of established nomenclature we shall refer to this method of searching for zeros of $F(x)$ as the Newton descent, and now motivate it by rederiving it from a minimization principle. Rewriting Eq. (5) in terms of a "cost function" $F(x)^{2}$,

$$
d \tau=-\frac{F^{\prime}(x)}{F(x)} d x=-\left(\frac{1}{2} \frac{d}{d x} \ln F(x)^{2}\right) d x
$$

and integrating,

$$
\tau=-\frac{1}{2} \int_{x(0)}^{x} d x^{\prime}\left(\frac{d}{d x} \ln F\left(x^{\prime}\right)^{2}\right)=-\frac{1}{2} \ln \frac{F(x)^{2}}{F(x(0))^{2}},
$$

we find that the deviation of $F(x)$ from $F\left(x^{*}\right)=0$ decays exponentially with the fictitious time,

$$
F(x(\tau))=F(x(0)) e^{-\tau},
$$

with the fixed point $x^{*}=\lim _{\tau \rightarrow \infty} x(\tau)$ reached at exponential rate. In other words, the Newton descent, derived here as an overcautious version of the damped Newton method, is a flow that minimizes the cost function $F(x)^{2}$.

\subsection{Multi-dimensional Newton Descent}

Due to the exponential divergence of nearby trajectories in chaotic dynamical systems, fixed point searches based on direct solution of the fixed-point condition Eq. (1) as an initial value problem can be numerically very unstable. Methods that start with initial guesses $\tilde{x}_{i}$ for a number of points along the cycle are considerably more robust and safer. Hence we consider next a set of periodic orbit conditions

$$
F_{i}(x)=x_{i}-f_{i}(x)=0, \quad x \in \mathbb{R}^{n d}
$$

where the periodic orbit traverses $n$ Poincaré sections (multipoint shooting method 78$), f_{i}(x)$ is the Poincaré return map from a section to the next one, and the index $i$ runs over $n d$ values, that is $d$ dimensions for each Poincaré section crossing. In this case the expansion Eq. (3) yields the Newton-Raphson iteration

$$
\tilde{x}_{k}^{(m+1)}=\tilde{x}_{k}^{(m)}-\left(\frac{\mathbf{1}}{\mathbf{1}-\mathbf{J}\left(\tilde{x}^{(m)}\right)}\right)_{k l} F_{l}\left(\tilde{x}^{(m)}\right), \quad \mathbf{J}(x)_{k l}=\frac{\partial f_{k}(x)}{\partial x_{l}},
$$

where $\mathbf{J}(x)$ is the $[d n \times d n]$ Jacobian matrix of the map $f(x)$, and $\tilde{x}^{(m)}$ is the $m$ th Newton-Raphson cycle point estimate.

The Newton descent method Eq. (5) now takes form

$$
\frac{\partial F_{i}(\tilde{x})}{\partial x_{j}} d x_{j}=-F_{i}(\tilde{x}) d \tau
$$

crete02: submitted to World Scientific on March 10, 2003 
Contracting both sides with $F_{i}(\tilde{x})$ and integrating, we find that

$$
F^{2}(\tilde{x})=\sum_{i=1}^{d n} F_{i}(\tilde{x})^{2}
$$

can be interpreted as the cost function Eq. (6), also decaying exponentially, $F^{2}(\tilde{x}(\tau))=F^{2}(\tilde{x}(0)) e^{-2 \tau}$, with the fictitious time gradient flow Eq. (9) now taking a multi-dimensional form:

$$
\frac{d \tilde{x}}{d \tau}=-\frac{1}{\mathbf{1}-\mathbf{J}(\tilde{x})} F(\tilde{x}) .
$$

Biham and collaborators 11 (see also Refs. 1213 ) were the first to introduce a fictitous time flow in searches for periodic orbits of low-dimensional maps, with a diagonal matrix with entries \pm 1 in place of the $1 /(\partial F / \partial x)$ matrix in Eq. (11). As we lack good visualizations of high-dimensional flows, for us the $\partial F / \partial x$ matrix is essential in determining the direction in which the cycle points should be adjusted.

Here we have considered the case of the guess $\tilde{x}$ a vector in a finite-dimensional vector space, with $F^{2}(\tilde{x})$ the penalty for the distance of $F(\tilde{x})$ from its zero value at a fixed point $x^{*}$. Our next task is to generalize the cost function to a cost functional $F^{2}[\tilde{x}]$ which measures the distance of a loop $\tilde{x}(s) \in L(\tau)$ from a periodic orbit $x(t) \in p$.

\section{Newton Descent in Loop Space}

For a flow described by a set of ODEs, multipoint shooting method of Sec. 2.2 can be quite efficient. However, multipoint shooting requires a set of phase space Poincaré sections such that an orbit leaving one section reaches the next one in a qualitatively predictable manner, without traversing other sections along the way. In turbulent, high-dimensional flows such sequences of sections are hard to come by. One cure for this ill might be a large set of Poincaré sections, with the intervening flight segments short and controllable. Here we shall take another path, and discard fixed Poincaré sections altogether.

Emboldened by success of methods such as the multipoint shooting (which eliminates the long-time exponential instability by splitting an orbit into a number of short segments, each with a controllable expansion rate) and the cyclist relaxation methods 8 (which replace map iteration by a contracting flow whose attractor is the desired periodic orbit of the original iterative dynamics), we now propose a method in which the initial guess is not a finite set of points, but an entire smooth, differentiable closed loop.

A general flow Eq. (2) has no extremal principle associated with it (we discuss the simplification of our method in the case of Hamiltonian mechanics in Sec. (4), so there is a great deal of arbitrariness in constructing a flow in a loop space. We shall introduce here the simplest cost function which penalizes mis-orientation of the local loop tangent vector $\tilde{v}(\tilde{x})$ relative to the dynamical velocity field $v(\tilde{x})$ of Eq. (2), and construct a flow in the loop space which minimizes this function. This flow is corralled by general topological features of the dynamics, with rather distant

crete02: submitted to World Scientific on March 10, 2003 
(c)

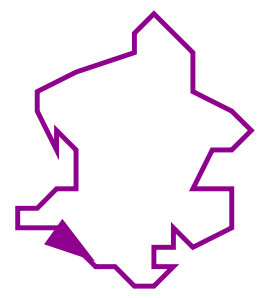

(b)

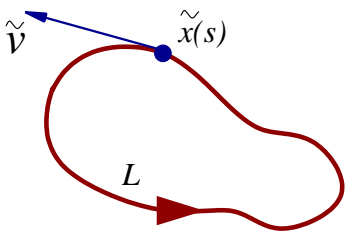

(c)

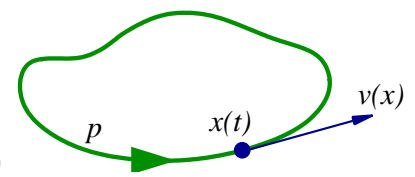

Figure 2. (a) A continuous path; (b) a loop $L$ with its tangent velocity vector $\tilde{v}$; (c) a periodic orbit $p$ defined by the vector field $v(x)$.

initial guesses converging to the desired orbit. Once the loop is sufficiently close to the periodic orbit, faster numerical algorithms can be employed to pin it down.

In order to set the notation, we shall distinguish between (see Fig. 2):

closed path: any closed (not necessarily differentiable) continuous curve $J \subset \mathcal{M}$.

loop: a smooth, differentiable closed curve $\tilde{x}(s) \in L \subset \mathcal{M}$, parametrized by $s \in$ $[0,2 \pi]$ with $\tilde{x}(s)=\tilde{x}(s+2 \pi)$, with the magnitude of the loop tangent vector fixed by the (so far arbitrary) parametrization of the loop,

$$
\tilde{v}(\tilde{x})=\frac{d \tilde{x}}{d s}, \quad \tilde{x}=\tilde{x}(s) \in L .
$$

annulus: a smooth, differentiable surface $\tilde{x}(s, \tau) \in L(\tau)$ swept by a family of loops $L(\tau)$, by integration along a fictitious time flow (see Fig. B (a))

$$
\dot{\tilde{x}}=\frac{\partial \tilde{x}}{\partial \tau} .
$$

periodic orbit: given a smooth vector field $v=v(x),(x, v) \in \mathbf{T} \mathcal{M}$, periodic orbit $x(t) \in p$ is a solution of

$$
\frac{d x}{d t}=v(x), \quad \text { such that } x(t)=x\left(t+T_{p}\right),
$$

where $T_{p}$ is the shortest period of $p$.

\subsection{Newton Descent in the Loop Space}

In the spirit of Eq. (10), we now define a cost functional for a loop and the associated fictitious time $\tau$ flow which sends an initial loop $L(0)$ via a loop family $L(\tau)$ into the periodic orbit $p=L(\infty)$, see Fig. 3 (a). The only thing that we are given is the velocity field $v(x)$, and we want to "comb" the loop $L(\tau)$ in such a way that its tangent field $\tilde{v}$ aligns with $v$ everywhere, see Fig. B(b). The simplest cost functional for the job is

$$
F^{2}[\tilde{x}]=\frac{1}{2 \pi} \oint_{L} d s(\tilde{v}-\lambda v)^{2}, \quad \tilde{v}=\tilde{v}(\tilde{x}(s, \tau)), \quad v=v(\tilde{x}(s, \tau)) .
$$

As we have fixed the loop period to $s=2 \pi$, the parameter $\lambda=\lambda(s, \tau)$ is needed to match the magnitude of the tangent field $\tilde{v}$ (measured in the loop parametrization 

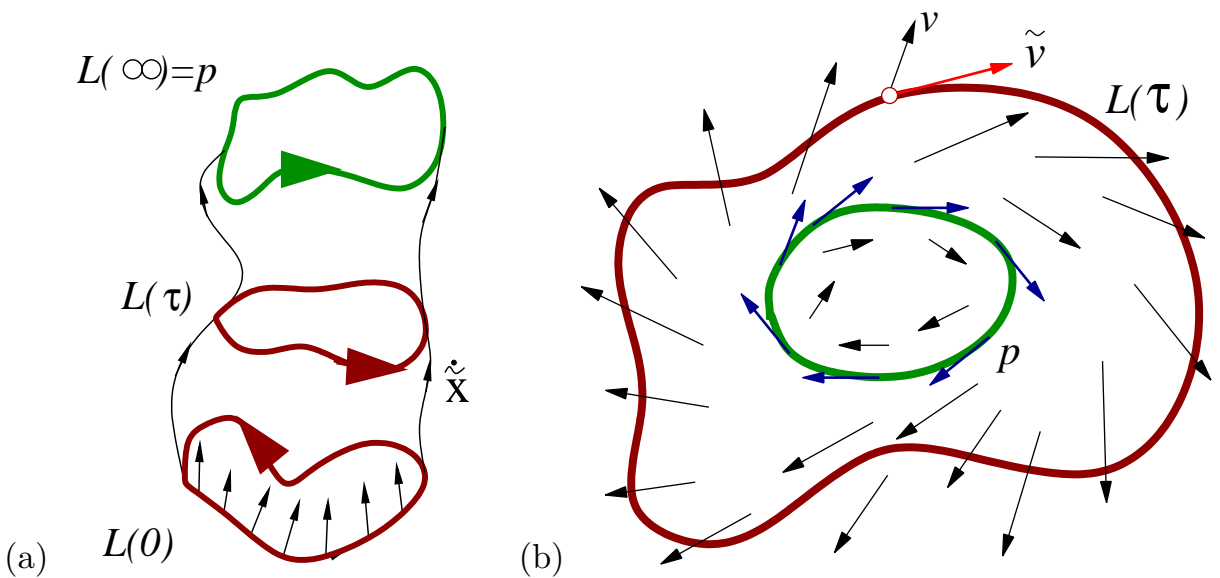

Figure 3. (a) An annulus $L(\tau)$ with vector field $\dot{\tilde{x}}$ connecting smoothly the initial loop $L(0)$ to a periodic orbit $p$. (b) In general the orientation of the loop tangent $\tilde{v}(\tilde{x})$ does not coincide with the orientation of the velocity field $v(\tilde{x})$; for a periodic orbit $p$ it does so at every $x \in p$.

units $s$ ) to the velocity field $v$ (measured in the dynamical time units $t$ ). $|v|$ cannot vanish anywhere along the loop $L(\tau)$, as in that case the loop would be passing through an equilibrium point, and have infinite period. We shall take a very simple choice, and set $\lambda$ to be a global, space-independent loop parameter $\lambda=\lambda(\tau)$. In the limit where the loop is the desired periodic orbit $p$, this $\lambda$ is the ratio of the dynamical period $T_{p}$ to the loop parametrization period $2 \pi, \lambda=T_{p} / 2 \pi$. More general choices of the parametrization $s$ will be discussed elsewhere. 14

Take a derivative of the cost functional $F^{2}[\tilde{x}]$ with respect to the (yet undetermined) fictitious time $\tau$,

$$
\frac{d F^{2}}{d \tau}=\frac{1}{\pi} \oint_{L} d s(\tilde{v}-\lambda v) \frac{d}{d \tau}(\tilde{v}-\lambda v) .
$$

The simplest, exponentially decreasing cost functional is obtained by taking the $\tilde{x}(s, \tau)$ dependence on $\tau$ to be point-wise proportional to the deviation of the two vector fields

$$
\frac{d}{d \tau}(\tilde{v}-\lambda v)=-(\tilde{v}-\lambda v)
$$

so the fictitious time flow drives the loop to $L(\infty)=p$, see Fig. 3 (a):

$$
\tilde{v}-\lambda v=\left.e^{-\tau}(\tilde{v}-\lambda v)\right|_{\tau=0} .
$$

Making the $\tilde{x}$ dependence in Eq. (13) explicit we obtain our main result, the Newton descent PDE which evolves the initial loop $L(0)$ into the desired periodic orbit $p$

$$
\frac{\partial^{2} \tilde{x}}{\partial s \partial \tau}-\lambda A \frac{\partial \tilde{x}}{\partial \tau}-\frac{\partial \lambda}{\partial \tau} v=\lambda v-\tilde{v}, \quad A_{i j}(x)=\frac{\partial v_{i}(x)}{\partial x_{j}}
$$

in the fictitious time $\tau \rightarrow \infty$. Here $A$ is the matrix of variations of the flow (its integral around $p$ yields the linearized stability matrix for the periodic orbit $p$ ).

crete02: submitted to World Scientific on March 10, 2003 


\subsection{Loop Initialization and Numerical Integration}

Replacement of a finite number of points along a trajectory by a closed smooth loop, and of the Newton-Raphson iteration by the Newton descent flow results in a second order PDE for the loop evolution. The loop parameter $s$ converges (up to a proportionality constant) to the dynamical time $t$ as the loop converges to the desired periodic orbit. The flow parameter $\tau$ plays the role of a fictitious time. Our aim is to apply this method to high-dimensional flows; and thus we have replaced the initial ODE dynamics Eq. (2) by a very high-dimensional PDE. And here our troubles start - can this be implemented at all? How do we get started?

A qualitative understanding of the dynamics is the essential prerequisite to successful periodic orbit searches. We start by long-time numerical runs of the dynamics, in order to get a feeling for frequently visited regions of the phase space ("natural measure"), and to search for close recurrences. We construct the initial loop $L(0)$ using the intuition so acquired. Taking a fast Fourier transform of the guess, keeping the lowest frequency components, and transforming back to the initial phase space helps smooth the initial loop $L(0)$. A simple linear stability analysis shows that the smoothness of the loop is maintained by the flow in the fictitious time $\tau$. This, as well as worries about the marginal stability eigenvalues and other details of the numerical integration of the loop flow Eq. (15), are described in Ref. 14. Suffice it to say that after a considerable amount of computation one is rewarded by periodic orbits that could not have been obtained by the methods employed previously.

\section{Extensions of the Method}

In classical mechanics particle trajectories are solutions of a different variational principle, the Hamilton's variational principle. For example, one can determine a periodic orbit of a billiard by wrapping around a rubber band of roughly correct topology, and then moving the points along the billiard walls until the length (that is, the action) of the rubber band is extremal (maximal or minimal under infinitesimal changes of the boundary points). In this case, extremization of action requires only $D$-dimensional ( $D=$ degrees of freedom) rather than $2 D$-dimensional $(2 D=$ dimension of the phase space) variations.

Can we exploit this fact to simplify our calculations in Newtonian mechanics? The answer is yes, and easiest to understand in terms of the Hamilton's variational principle which states that classical trajectories are extrema of the Hamilton's principal function (or, for fixed energy, the action)

$$
R\left(q_{1}, t_{1} ; q_{0}, t_{0}\right)=\int_{t_{0}}^{t_{1}} d t \mathcal{L}(q(t), \dot{q}(t), t),
$$

where $\mathcal{L}(q, \dot{q}, t)$ is the Lagrangian. Given a loop $L(\tau)$ we can compute not only the tangent "velocity" vector $\tilde{v}$, but also the local loop "acceleration" vector

$$
\tilde{a}=\frac{d^{2} \tilde{x}}{d s^{2}}
$$

crete02: submitted to World Scientific on March 10, 2003 
and indeed, as many $s$ derivatives as needed. Matching the dynamical acceleration $a(\tilde{x})$ with the loop "acceleration" $\tilde{a}(\tilde{x})$ results in an equation for the evolution of the loop

$$
\frac{d}{d \tau}\left(\tilde{a}-\lambda^{2} a\right)=-\left(\tilde{a}-\lambda^{2} a\right),
$$

where $\lambda^{2}$ appears instead of $\lambda$ for dimensional reasons. This equation can be reexpressed in terms of loop variables $\tilde{x}(s)$; the resulting equation is somewhat more complicated than Eq. (15), but the saving is significant - only $1 / 2$ of the phase-space variables appears in the fictitious time flow. More generally, the method works for extremization of functions of form $\mathcal{L}(q, \dot{q}, \ddot{q}, \ldots, t)$, with considerable computational savings. 14

\section{Applications}

We now offer several examples of the application of the Newton descent in the loop space, Eq. (15).

\subsection{Unstable Recurrent Patterns in a Classical Field Theory}

One of the simplest and extensively studied spatially extended dynamical systems is the Kuramoto-Sivashinsky system 17

$$
u_{t}=\left(u^{2}\right)_{x}-u_{x x}-\nu u_{x x x x}
$$

which arises as an amplitude equation for interfacial instabilities in a variety of contexts. The "flame front" $u(x, t)$ has compact support, with $x \in[0,2 \pi]$ a periodic space coordinate. The $u^{2}$ term makes this a nonlinear system, $t$ is the time, and $\nu$ is a fourth-order "viscosity" damping parameter that irons out any sharp features. Numerical simulations demonstrate that as the viscosity decreases (or the size of the system increases), the "flame front" becomes increasingly unstable and turbulent. 1819 The task of the theory is to describe this spatio-temporal turbulence and yield quantitative predictions for its measurable consequences.

As was argued in Ref. 20 , turbulent dynamics of such systems can be visualized as a walk through the space of unstable spatio-temporally recurrent patterns. In the PDE case we can think of a spatio-temporally discretized guess solution as a surface covered with small but misaligned tiles. Decreasing Eq. (12) by the Newton descent means smoothing these strutting fish scales into a smooth surface, a solution of the $\mathrm{PDE}$ in question.

In case at hand it is more convenient to transform the problem to Fourier space. If we impose the periodic boundary condition $u(t, x+2 \pi)=u(t, x)$ and choose to study only the odd solutions $u(-x, t)=-u(x, t), 20$ the spatial Fourier series for the wavefront is

$$
u(x, t)=i \sum_{k=-\infty}^{\infty} a_{k}(t) \exp (i k t)
$$


(a)

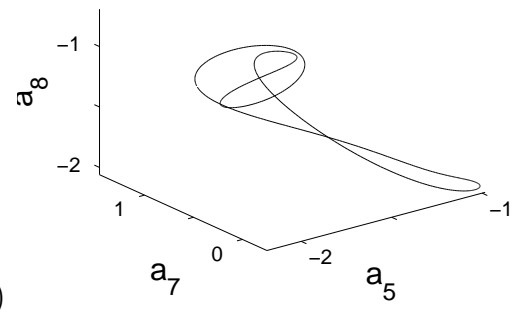

(b)

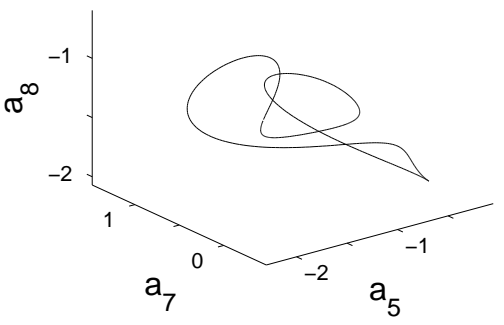

Figure 4. (a) An initial guess $L(0)$, and (b) the periodic orbit $p$ of period $T_{p}=0.5051$ reached by the Newton descent, the Kuramoto-Sivashinsky system in a spatio-temporally turbulent regime (viscosity parameter $\nu=0.01500, d=32$ Fourier modes truncation). In discretization of the initial loop $L(0)$ each point has to be specified in all $d$ dimensions; here the coordinates $\left\{a_{5}, a_{7}, a_{8}\right\}$ are picked arbitrarily, other projections from $d=32$ dimensions to a subset of 3 coordinates are equally (un)informative.

with real Fourier coefficients $a_{-k}=-a_{k}$, and Eq. (16) takes form

$$
\dot{a_{k}}=\left(k^{2}-\nu k^{4}\right) a_{k}-k \sum_{m=-\infty}^{\infty} a_{m} a_{k-m} .
$$

After the initial transients die out, for large $k$ the magnitude of $a_{k}$ Fourier component decreases exponentially with $k^{4}$, justifying use of Galerkin truncations in numerical simulations. As in numerical work on any PDE we thus replace Eq. (16) by a finite but high-dimensional system of ODEs. The initial searches for the unstable recurrent patterns for this spatially extended system found several hundreds of periodic solutions close to the onset of spatiotemporal chaos, but a systematic exploration of more turbulent regimes was unattainable by the numerical techniques employed. 20121

With decreasing viscosity $\nu$ the system becomes quite turbulent, with the spatiotemporal portraits of the flame front $u(x, t)$ a complex labyrinth of eddies of different scales and orientations, and its Fourier space dynamics Eq. (18) a complicated high-dimensional trajectory.

In Fig. 4 we give an example of a Newton descent calculation for this system, for the viscosity parameter $\nu$ significantly lower than in the earlier investigations. Although the initial guess $L(0)$ is quite far from the final configuration $p=L(\infty)$, the method succeeds in molding the starting loop into a periodic solution of this high dimensional flow. A systematic exploration of the shortest cycles found, and the hierarchy of longer cycles will be reported elsewhere. 22

\subsection{Hénon-Heiles and Restricted 3-body Problems}

Next we offer two examples of the applicability of the extension of the Newton descent of Sec. 4 to low-dimensional Hamiltonian flows.

crete02: submitted to World Scientific on March 10, 2003 

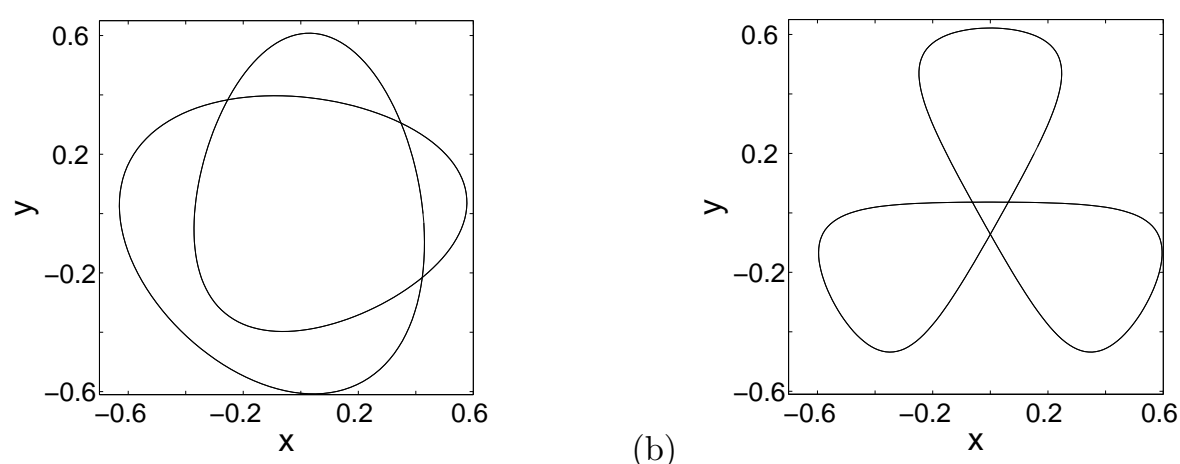

Figure 5. (a) An initial loop $L(0)$, and (b) the periodic orbit $p$ reached by the Newton descent, the Hénon-Heiles system in a chaotic regime, $E=0.1794$. The period was fixed arbitrarily to $T=13.1947$ by taking a fixed value of the scaling $\lambda=2.1$.

Hénon-Heiles Hamiltonian 23

$$
H=\frac{1}{2}\left(\dot{x}^{2}+\dot{y}^{2}+x^{2}+y^{2}\right)+x^{2} y-\frac{y^{3}}{3}
$$

is frequently used in astrophysics. Fig. [5 shows an application of the method of Sec. 4 to a periodic orbit search restricted to the configuration space.

In the Hénon-Heiles case the acceleration $\left(a_{x}, a_{y}\right)$ depends only on the configuration coordinates $(x, y)$. More generally, the $a$ 's could also depend on $(\dot{x}, \dot{y})$, as is the case for the restricted three-body problem equations of motion 24

$$
\begin{aligned}
\ddot{x} & =2 \dot{y}+x-(1-\mu) \frac{x+\mu}{r_{1}^{3}}-\mu \frac{x-1+\mu}{r_{2}^{3}} \\
\ddot{y} & =-2 \dot{x}+y-(1-\mu) \frac{y}{r_{1}^{3}}-\mu \frac{y}{r_{2}^{3}} \\
r_{1} & =\sqrt{(x+\mu)^{2}+y^{2}}, \quad r_{2}=\sqrt{(x-1+\mu)^{2}+y^{2}}
\end{aligned}
$$

which describe the motion of a "test particle" in a rotating frame under the influence of the gravitational force of two heavy bodies with masses 1 and $\mu \ll 1$, fixed at $(-\mu, 0)$ and $(1-\mu, 0)$ in the $(x, y)$ coordinate frame. The periodic solutions of Eq. (20) correspond to periodic or quasi-periodic motion of the test particle in the inertial frame. Fig. 6] shows an application of the Newton descent method to this problem.

\section{Summary and Future Directions}

The periodic orbit theory approach to classically turbulent field theory is to visualize turbulence as a sequence of near recurrences in a repertoire of unstable spatiotemporal patterns. So far, existence of a hierarchy of spatio-temporally periodic solutions, and applicability of the periodic orbit theory in evaluation of global averages for spatially extended nonlinear system has been demonstrated in one example, the Kuramoto-Sivashinsky system. 17 The parameter ranges previously

crete02: submitted to World Scientific on March 10, 2003 


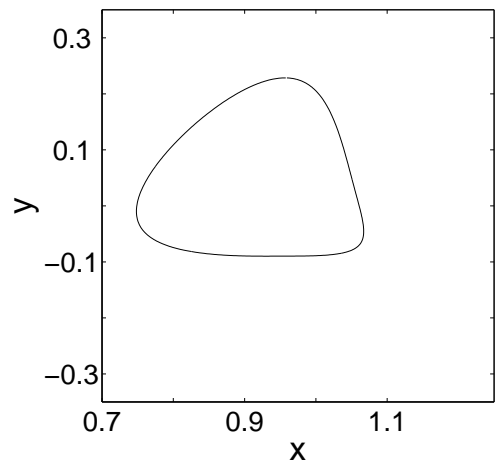

(b)

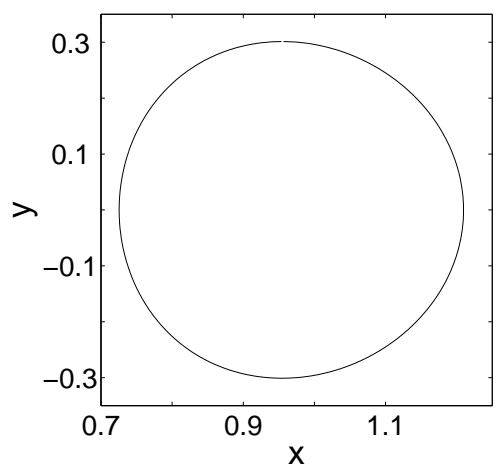

Figure 6. (a) An initial loop $L(0)$, and (b) the periodic orbit $p$ reached by the Newton descent, the restricted three body problem in the chaotic regime, $\mu=0.04, T_{p}=2.7365$.

explored probe the weakest nontrivial "turbulence", and it is an open question to what extent the approach remains implementable as such classical fields go more turbulent. Kawahara and Kida 25 were able to find two periodic solutions in a turbulent plane Couette flow, in a 15,422-dimensional discretization of the full 3-d Navier-Stokes equations, so we remain optimistic.

The bottleneck has been the lack of methods for finding even the simplest periodic orbits in high-dimensional flows, and the lack of intuition as to what such orbits would look like. Here we have formulated the "Newton descent" method, a very conservative method which emphasizes topological robustness at a considerable cost to numerical speed, and demonstrated that the method enables us to find the shortest spatio-temporally unstable periodic solutions of an (infinite dimensional) classical field theory, as well as several Hamiltonian flows.

In devising the Newton descent method we have made a series of restrictive choices, many of which could be profitably relaxed.

The choice of a Euclidean metric cost function $F^{2}[\tilde{x}]$ has no compelling merit, other than notational simplicity. For a flow like the Kuramoto-Sivashinsky the $a_{1}, a_{2}, \ldots$ directions are clearly more important than $a_{k}, a_{k+1}, \ldots, k$ large, and that is not accounted for by the current form of the cost function. A more inspired choice would use intrinsic information about dynamics, replacing $\delta_{i j} F_{i} F_{j}$ by a more appropriate metric $g_{i j} F_{i} F_{j}$ that penalizes straying away in the unstable directions more than deviations in the strongly contracting ones.

Loop parametrization. Once it is understood that given a vector field $v(x)$, the objective is to determine a loop $L(\infty)=p$ whose tangent vectors point along $v(x)$ everywhere along the loop, there is no reason to fix the loop parameter $s$ by making it proportional to the dynamical system time $t$. Any loop parametrization $s$ will do, and other choice might be more effective for numerical discretizations.

Zero modes. In numerical calculations we eliminate the marginal eigendirection along the loop by "gauge fixing", fixing one point on the loop by an arbitrary Poincaré section. This seems superfluous and perhaps should be eliminated in favor of some other, more invariant criterion.

crete02: submitted to World Scientific on March 10, 2003 
The Newton descent method introduced here replaces the Newton-Raphson iteration by an exponentially contracting flow. Keeping the fictitious time step $d \tau$ infinitesimal is both against the spirit of the Newton method, and not what we do in practice; once the approximate loop is sufficiently close to the desired periodic orbit, we replace $d \tau$ by discrete steps of increasing size $d \tau \rightarrow 1$, in order to regain the super-exponential convergence of the Newton method.

Topology. As for high-dimensional flows we are usually clueless as to what the solutions should look like, currently we have no way of telling to which periodic orbit the loop space flow Eq. (15) will take our initial guess, other than to the "nearest" periodic orbit of topology "similar" to the initial loop.

\section{References}

1. M.C. Gutzwiller, Chaos in Classical and Quantum Mechanics (Springer-Verlag, New York 1990).

2. P. Cvitanović, Physica A 288, 61 (2000); nlin.CD/0001034

3. E. Hopf, Bereich. Sächs. Acad. Wiss. Leipzig, Math. Phys. Kl. 94, 19 (1942).

4. D.W. Moore and E.A. Spiegel, Astrophys. J. 143, 871 (1966).

5. N.H. Baker, D.W. Moore and E.A. Spiegel, Quatr. J. Mech. and Appl. Math. 24, 391 (1971).

6. E.A. Spiegel, Proc. Roy. Soc. A413, 87 (1987).

7. J. Stoer and R. Bulirsch, Introduction to Numerical Analysis (Springer-Verlag, New York 1983).

8. P. Cvitanović et al, Classical and Quantum Chaos (Niels Bohr Institute, Copenhagen, 2003); www.nbi.dk/ChaosBook.

9. V.I. Arnol'd, Ordinary Differential Equations (Springer-Verlag, New York 1992).

10. W.H. Press, S.A. Teukolsky, W.T. Vetterling and B.P. Flannery, Numerical Recipes in $C$ (Cambridge University Press, 1992).

11. O. Biham and W. Wenzel, Phys. Rev. Lett. 63, 819 (1989); Phys. Rev. A 42, 4639 (1990).

12. P. Schmelcher and F.K. Diakonos, Phys. Rev. Lett. 78, 4733 (1997); Phys. Rev. E 57, 2739 (1998); F. K. Diakonos, P. Schmelcher, O. Biham, Phys. Rev. Lett. 81, 4349 (1998); D. Pingel, P. Schmelcher and F.K. Diakonos, O. Biham, Phys. Rev. E 64, 026214 (2001).

13. C. Chandre, F.K. Diakonos and P. Schmelcher, section "Discrete cyclist relaxation method", in Ref. 8 .

14. Y. Lan and P. Cvitanović, A variational method for finding periodic orbits (in preparation).

15. J.W. Thomas, Numerical Partial Differential Equations (Springer-Verlag, New York 1995).

16. D. Auerbach, P. Cvitanović, J.-P. Eckmann, G.H. Gunaratne and I. Procaccia, Phys. Rev. Lett. 58, 2387 (1987).

17. Y. Kuramoto and T. Tsuzuki, Progr. Theor. Physics 55, 365 (1976);

G.I. Sivashinsky, Acta Astr. 4, 1177 (1977).

crete02: submitted to World Scientific on March 10, 2003 
18. I.G. Kevrekidis, B. Nicolaenko and J.C. Scovel, SIAM J. Applied Math. 50, 760 (1990).

19. C. Foias, B. Nicolaenko, G.R. Sell and R. Témam, J. Math. Pures et Appl. 67, 197 (1988).

20. F. Christiansen, P. Cvitanović and V. Putkaradze, Nonlinearity 10, 55 (1997); chao-dyn/9606016

21. S.M. Zoldi and H.S. Greenside, Phys. Rev. E 57, R2511 (1998).

22. Y. Lan, Periodic orbit theory of spatiotemporal chaos, Ph.D Thesis (School of Physics, Georgia Tech, 2004).

23. M. Hénon and C. Heiles, Astron. J. 69, 73 (1964).

24. V. Szebehely, Theory of Orbits (Academic Press, New York 1967).

25. G. Kawahara and S. Kida, J. Fluid Mech. 449, 291 (2001).

crete02: submitted to World Scientific on March 10, 2003 